\title{
La comunicación como estrategia generadora de confianza en los emprendimientos
}

\section{Communication as a confidence generating strategy in the enterprises}

Lizbeth Suarez Morales

Universidad Internacional del Ecuador, Ecuador

Jenny Maldonado Castro

Universidad Técnica Estatal de Quevedo, Ecuador

Autor para correspondencia: 1suarez@uide.edu.ec

Fecha de recepción: 05 de Agosto 2017 - Fecha de aceptación: 15 de Enero de 2018

Resumen: La comunicación es una estrategia generadora de confianza en los emprendimientos, por la capacidad de influencia en los individuos y la capacidad de generar y mantener la confianza, así como de perderla, cuando hay incongruencias entre lo se dice y hace, o cuando los mensajes se perciben como promesas inalcanzables o con tintes manipuladores. La comunicación es un proceso propio del ser humano en el que interactúan diferentes actores, la estructura social da paso a diversos tipos de comunicación. Los emprendimientos son organizaciones con capacidades de adaptación inmediata, para responder a las necesidades cambiantes de los diversos entornos, el objetivo de la investigación es "Analizar la comunicación como estrategia generadora de confianza en los emprendimientos." Para abordar el tema se han desarrollado tres fases, metodología, desarrollo y aspectos conclusivos. En la primera se han seleccionado bases de datos, búsqueda por palabras clave, análisis de abstract y selección de tres artículos de relación con el tema. En la segunda que corresponde al desarrollo, se planteó el emprendimiento desde la conceptualización de algunos autores relacionados a este campo de conocimiento así como la descripción de este sector de la economía en el Ecuador y las acciones gubernamentales para gestionarlo, luego se abordó la comunicación desde la perspectiva sicológica cognitiva en la efectividad de la comunicación y la correlación existente entre ellas. Finalmente se analizó la confianza como un elemento de integración social que determina el procedimiento de las personas en actividades, especialmente en el trabajo, que la autora citada propone estrategias de comunicación para generar confianza.

Palabras clave: comunicación; emprendimiento; estrategia; confianza

Abstract: Communication is a strategy that generates confidence in entrepreneurship, the capacity to influence individuals and the ability to generate and maintain trust, as well as losing it, when there is inconsistency between what is said and done, or when messages are communicated. Perceived as unattainable promises or manipulative dyes. Communication is a process of the human being in which different actors interact, the social structure gives way to various types of communication. The enterprises are organizations with immediate adaptation capabilities, to respond to the changing needs of different environments, the objective of the research is "Analyze communication as a strategy that generates confidence in the enterprises." Three phases, methodology, development and conclusive aspects have been developed to address the theme. In the first one, we have selected databases, keyword search, abstract analysis and selection of three articles related to the topic. In the second that corresponds to the development, the entrepreneurship was considered from the conceptualization of 
some authors related to this field of knowledge as well as the description of this sector of the economy in Ecuador and the governmental actions to manage it, then the communication since The cognitive psychological perspective on the effectiveness of communication and the correlation between them. Finally, trust was analyzed as an element of social integration that determines the procedure of the people in activities, especially in the work, that the mentioned author proposes communication strategies to generate confidence.

Key words: communication; entrepreneurship; strategy; confidence

\section{Introducción}

La comunicación es un proceso propio del ser humano en el que interactúan diferentes actores, en la estructura social se da paso a diversos tipos de comunicación. Estas tipologías tienen como elemento transversal a la confianza, que influye en el rol del individuo y ciudadano.

En la teorías de la elección racional se define a la confianza como el conjunto de expectativas racionales, basadas en la comprensión del interés del otro con respecto al propio y en cálculos que sopesan los costos y beneficios de ciertos cursos de acción de quien confía o de la persona en quien se confía. La comunicación, está integrada por factores que generan confianza y explican el comportamiento en contextos donde el intercambio se gestiona, uno de estos elementos se denomina efectividad de la comunicación.

\section{Metodología}

La metodología utilizada en este trabajo es descriptivo a través del análisis bibliográfico de bases de datos, de las cuales se seleccionaron cuatro, en las que se utilizó palabras clave de búsqueda "comunicación y confianza", de aquella búsqueda en total se encontraron 3831 artículos relacionados con la temática de investigación, que luego del análisis de contenido, se han identificado 6 publicaciones académicas en directa relación a la tema, subtema y título de este escrito. En la siguiente tabla se evidencian las bases de datos investigadas y los resultados obtenidos de esta búsqueda.

Tabla 1.

\begin{tabular}{lllc}
\hline Nombre & Palabras Clave & $\begin{array}{c}\text { Resultado de la búsqueda } \\
\text { (número de artículos) }\end{array}$ & $\begin{array}{c}\text { Artículos } \\
\text { Validos }\end{array}$ \\
\hline Dialnet & comunicación y confianza & 570 & 3 \\
ScienceDirect & comunicación y confianza & 3195 & 0 \\
Scopus & comunicación y confianza & 4 & 1 \\
Ebesco & comunicación y confianza & 62 & 2 \\
& TOTAL & $\mathbf{3 8 3 1}$ & $\mathbf{6}$ \\
\hline
\end{tabular}

Luego de identificados los artículos, se analizó el abstract, la pregunta de investigación o hipótesis si la investigación lo amerita, el marco teórico, el análisis de los datos, las relaciones teóricas y las conclusiones, según se denota en la tabla 2.

Tabla 2. 


\begin{tabular}{|c|c|c|c|c|}
\hline $\begin{array}{c}\text { Título de } \\
\text { Artículo } \\
\end{array}$ & $\begin{array}{l}\text { Objetivo de } \\
\text { Artículo } \\
\end{array}$ & $\begin{array}{c}\text { Preguntas de } \\
\text { Investigación }\end{array}$ & $\begin{array}{c}\text { Hipótesis de } \\
\text { Investigación }\end{array}$ & Método de Investigación \\
\hline $\begin{array}{l}\text { Estrategias de } \\
\text { comunicación } \\
\text { para generar } \\
\text { confianza }\end{array}$ & \begin{tabular}{l}
\multicolumn{1}{c}{ Proponer } \\
estrategias de \\
comunicación \\
adaptadas al \\
objetivo \\
actitudinal de \\
recuperar la \\
Confianza, para \\
lo que como \\
objetivo previo \\
se necesita \\
conocer en \\
profundidad el \\
término.
\end{tabular} & $\begin{array}{l}\text { ¿Es la Confianza } \\
\text { una actitud del } \\
\text { individuo que } \\
\text { requiere } \\
\text { estrategias } \\
\text { específicas de } \\
\text { comunicación? } \\
\text { ¿Qué diferencias y } \\
\text { similitudes tiene } \\
\text { con otros } \\
\text { intangibles como } \\
\text { la imagen y la } \\
\text { reputación? } \\
\text { ¿Cuáles son las } \\
\text { estrategias de } \\
\text { comunicación más } \\
\text { adecuadas? }\end{array}$ & & $\begin{array}{l}\text { Método descriptivo a través } \\
\text { de un estudio bibliográfico del } \\
\text { concepto de Confianza como } \\
\text { actitud, tomando como } \\
\text { referencia la Psicología social, } \\
\text { cognitiva y la Psicología del } \\
\text { consumidor, así como la } \\
\text { referencia a bibliografía sobre } \\
\text { comunicación, relaciones } \\
\text { públicas y estrategia. }\end{array}$ \\
\hline $\begin{array}{l}\text { La generación } \\
\text { de la Confianza } \\
\text { y sus efectos } \\
\text { sobre el } \\
\text { comportamiento }\end{array}$ & $\begin{array}{l}\text { Analizar la } \\
\text { relación } \\
\text { interpersonal } \\
\text { entre el docente y } \\
\text { subordinado } \\
\text { desde los } \\
\text { diferentes } \\
\text { factores y su } \\
\text { relación con la } \\
\text { confianza. }\end{array}$ & $\begin{array}{l}\text { Factores que } \\
\text { generan confianza } \\
\text { y explican el } \\
\text { comportamiento } \\
\text { en contextos donde } \\
\text { el intercambio es } \\
\text { esencialmente } \\
\text { económico ¿se } \\
\text { comportan de igual } \\
\text { forma cuando el } \\
\text { intercambio de } \\
\text { naturaleza es } \\
\text { social? }\end{array}$ & $\begin{array}{l}\text { H1. En un } \\
\text { contexto de } \\
\text { intercambio social } \\
\text { como el que tiene } \\
\text { lugar en la } \\
\text { universidad se da } \\
\text { mucha relación } \\
\text { positiva entre la } \\
\text { efectividad de la } \\
\text { comunicación } \\
\text { eficiente entre las } \\
\text { partes y el nivel de } \\
\text { confianza } \\
\text { establecida entre } \\
\text { ellos. (Se tomó } \\
\text { solo la primera } \\
\text { hipótesis y su } \\
\text { análisis por la } \\
\text { interrelación con el } \\
\text { tema de } \\
\text { investigación } \\
\text { propuesto.) }\end{array}$ & $\begin{array}{l}\text { Es una investigación empírica, } \\
\text { cuantitativa, por la autonomía } \\
\text { con la que se llevan a cabo las } \\
\text { relaciones interpersonales de } \\
\text { dependencia en la Universidad. } \\
\text { Se seleccionó una muestra de } \\
\text { un censo de población, se } \\
\text { realizó un análisis factorial } \\
\text { confirmatoria y modelo de } \\
\text { estructuras de covarianza. }\end{array}$ \\
\hline $\begin{array}{l}\text { La dinámica de } \\
\text { la confianza: } \\
\text { Comunicación, } \\
\text { acción y terceras } \\
\text { partes }\end{array}$ & $\begin{array}{l}\quad \text { Analizar la } \\
\text { confianza en los } \\
\text { individuos, } \\
\text { organizaciones, } \\
\text { instituciones y } \\
\text { sistemas. }\end{array}$ & $\begin{array}{l}\text { ¿Qué es la } \\
\text { confianza, en } \\
\text { quién se puede } \\
\text { confiar, en qué y } \\
\text { con qué bases? }\end{array}$ & & $\begin{array}{l}\text { Método el estudio } \\
\text { bibliográfico del concepto de } \\
\text { Confianza, Comunicación y sus } \\
\text { aplicaciones e implicaciones } \\
\text { sociales. }\end{array}$ \\
\hline
\end{tabular}

Fuente: Investigación bibliográfica, Adaptación propia

\section{Emprendimiento}

\section{Resultados y discusión}

El emprendimiento es una forma de desarrollo de las economías, sensible de ser estudiado desde varias perspectivas, a nivel mundial, esta temática, se ha convertido en un fenómeno de 
desarrollo económico y social que permite la inserción de los individuos en la actividad económica (Drakopoulou Dodd \& Hynes, 2011).

El gestor del emprendimiento es el emprendedor; para Schumpeter (1950, pág. 116), es "la innovación que se desarrolla a partir de la capacidad para emprender, por tanto, los emprendedores no son necesariamente capitalistas, administradores o inventores, ya que finalmente se trata de personas con una capacidad para "combinar" los factores de producción existentes y obtener mejores resultados de dicha forma de utilizarlos, es decir, de innovar."

La visión de, (Schumpeter, 1950) respecto al emprendedor, lo denota como una fuente de investigación desde la perspectiva de la capacidad del sujeto que combina los factores de producción y la innovación para mejorar la capacidad de generación e impacto desestabilizador en la economía.

Por otra parte Rodríguez (2009), manifiesta que el concepto de emprendedor se caracterizaba por dos tipos de individuos: el guerrero arriesgado que emprendía una lucha o hazaña, reconocido por sus características personales; y el rey o jefe de Estado que planeaba sus estrategias y políticas para obtener con éxito sus metas, y era reconocido por sus funciones.

Adicionalmente (Rodríguez, 2009), basado en (Casson, 2005) propone dos caminos similares de características personales y funcionales para definir el concepto de emprendedor, con base en la economía, diferenciando al emprendedor del inversionista.

En Ecuador se ha desarrollado en un ambiente desarticulado (Claudia Eugenia Toca Torres, 2017), para el emprendimiento, de acciones aisladas en los organismos gubernamentales y no gubernamentales, por esta razón el gobierno central del Ecuador en la búsqueda de equiparar las condiciones de acceso al emprendimiento urbano y rural, ha impulsado programas de gestión de emprendimiento, que permita a los ciudadanos crear negocios con potencial de crecimiento que sean innovadores o altamente diferenciados. Es primordial manifestar que desde el 2007, la política económica del Ecuador cambia de rumbo a través de una nueva propuesta de carta magna en la que incluyeron y denotaron sectores antes olvidados.

Esta nueva Constitución aprobada en el 2008, se definió las condiciones necesarias para asegurar el desarrollo empresarial en el Ecuador, así como a través de Secretaria Nacional de Planificación (Senescyt, 2010), se instrumenta como política de Estado el Plan del Buen Vivir (2008-2013), y que hoy se ejecuta el Plan (2013-2017), que en el Objetivo No. 6 queda especificado: Garantizar el trabajo estable, justo y digno; y en la política 6.2 Promocionar emprendimientos exitosos, generadores de empleo.

En perspectiva de acondicionar un escenario adecuado para desarrollar emprendimientos, el gobierno del Ecuador incorporó las Finanzas Populares y Solidarias en su Constitución 2008, conforme establecen los artículos 308 al 312.

Por otra parte el (GEM, 2013) con su sede en Ecuador, y los estudios realizados en varios países del Mundo, manifiestan que el Ecuador consta entre los 15 países con mayor tendencia a iniciar nuevos negocios. Las fortalezas de sus emprendedores son la juventud y la 
capacidad de detectar oportunidades del mercado; sus debilidades, la falta de capital y la poca capacitación. El emprendimiento puede ser un motor de la economía, pero su impacto aún es incipiente.

Según el estudio, el Índice de Actividad Emprendedora Temprana, conocido como TEA, es del 15,82\% en Ecuador. Esto quiere decir que siete de cada 10 ecuatorianos adultos están en proceso de iniciar un negocio o gestionando una nueva empresa (de no más de 42 meses). Pese al dinamismo que evidencian los números, este índice considerado el eje del GEM, ha registrado un importante descenso respecto del 2004, la primera vez que se aplicó el estudio localmente, cuando el TEA fue del 27,2\%, y ubicó al Ecuador en el tercer lugar del ranking mundial. (GEM, 2013)

La investigación demuestra, que los emprendedores ecuatorianos que buscan una oportunidad de mercado, superan en el doble a aquellos que emprenden por necesidad; de este segmento, más de la mitad busca mayor independencia y el incremento de ingresos. Además, buena parte de estos nuevos negocios nacen en el sector de servicios al consumidor y se enfocan principalmente en el mercado doméstico.(GEM, 2013)

Más de la mitad de los emprendedores ecuatorianos están entre los 25 y 44 años de edad y la incorporación de mujeres a este segmento es cada vez más significativa. La diferencia radica en que los hombres emprenden primordialmente por oportunidad, mientras que las mujeres son más sensibles a la necesidad. Esta motivación usualmente es más determinante en segmentos que no han tenido la oportunidad de acceder a educación formal o que han completado un nivel medio. (GEM, 2013)

Uno de los principales obstáculos que señalan los emprendedores ecuatorianos, es la dificultad de acceso a fuentes de financiamiento. Por ello, un $84 \%$ de ellos inician sus negocios con capitales inferiores a los US\$ 10.000 , en gran medida provistos por familiares, amigos y conocidos. Uno de los efectos directos de esta limitación de recursos es que la mayoría de emprendimientos nace y depende en sus primeros años de tecnología antigua.(GEM, 2013)

Ecuador se inscribe en un listado que lo ubica entre los 15 países con mayor tendencia a iniciar nuevos negocios. Las fortalezas de sus emprendedores son la juventud y la capacidad de detectar oportunidades del mercado; sus debilidades, la falta de capital y la poca capacitación. El emprendimiento puede ser un motor de la economía, pero su impacto aún es incipiente, por las condiciones en las que surge.

Quiero ser mi propio jefe', 'necesito ganar más', 'se me ocurrió una idea genial'... son algunas de las razones por las que los ecuatorianos deciden arrancar un negocio propio, estas motivaciones han resultado lo suficientemente poderosas como para que el Ecuador sea considerado entre los países más emprendedores del mundo. (GEM, 2013)

Según el estudio, el Índice de Actividad Emprendedora Temprana, conocido como TEA, es del 15,82\% en Ecuador. Esto quiere decir que siete de cada 10 ecuatorianos adultos están en proceso de iniciar un negocio o gestionando una nueva empresa (de no más de 42 meses). Pese al dinamismo que evidencian los números, este índice considerado el eje del GEM ha registrado un 
importante descenso respecto del 2004, la primera vez que se aplicó el estudio localmente, cuando el TEA fue del 27,2\%, y ubicó al Ecuador en el tercer lugar del ranking mundial.(GEM, 2013)

Este retroceso no necesariamente es una mala noticia, sino el resultado de cambios que vale la pena analizar en sus respectivos contextos. En el concierto internacional, por ejemplo, el GEM demostró la tendencia mundial a la desaceleración por efecto de la crisis financiera desatada en 2008. De ahí que dos tercios del universo de países encuestados registraron descensos en sus índices ante el creciente pesimismo de hallar nuevas oportunidades por parte de los potenciales emprendedores.(GEM, 2013)

Una vía de aproximación para el análisis del emprendimiento son las motivaciones. La investigación demuestra que los emprendedores ecuatorianos que buscan una oportunidad de mercado, doblan a aquellos que emprenden por necesidad; de este segmento, más de la mitad busca mayor independencia y el incremento de ingresos. Además, buena parte de estos nuevos negocios nacen en el sector de servicios al consumidor y se enfocan principalmente en el mercado doméstico.(GEM, 2013)

Más de la mitad de los emprendedores ecuatorianos están entre los 25 y 44 años de edad y la incorporación de mujeres a este segmento es cada vez más significativa. La diferencia radica en que los hombres emprenden primordialmente por oportunidad, mientras que las mujeres son más sensibles a la necesidad. Esta motivación usualmente es más determinante en segmentos que no han tenido la oportunidad de acceder a educación formal o que han completado un nivel medio. Uno de los principales obstáculos que señalan los emprendedores ecuatorianos es la dificultad de acceso a fuentes de financiamiento. Por ello, un $84 \%$ de ellos inician sus negocios con capitales inferiores a los US\$10.000, en gran medida provistos por familiares, amigos y conocidos. Uno de los efectos directos de esta limitación de recursos es que la mayoría de emprendimientos nace y depende en sus primeros años de tecnología antigua.

Otra de las particularidades del GEM, (2013) es que intenta descubrir las relaciones relevantes entre el emprendimiento y el desarrollo de las economías locales. Con este propósito, un indicador clave es el número de empleos generados por estos nuevos emprendimientos y sus expectativas de expansión en el corto plazo. En el caso del Ecuador, esta información es preocupante, pues más del $90 \%$ de los emprendimientos genera solamente entre una y 5 plazas de empleo y sus previsiones para los cinco años siguientes se mantienen en los mismos niveles. En consecuencia, el impacto del emprendimiento en el desarrollo económico local por el momento es muy bajo aún.

El GEM ( 2013) divide a los países en tres grupos, según sus etapas de desarrollo, el primero: Economías basadas en factores productivos, el segundo: Economías basadas en eficiencia y el tercero: Economías basadas en innovación. Ecuador pertenece al segundo grupo junto a otros 21 países, entro los cuales constan varios latinoamericanos como Brasil, Chile Colombia, Perú, Argentina, Panamá, República Dominicana y Uruguay. Ecuador registra casi cuatro puntos porcentuales arriba del promedio del Índice de Actividad Emprendedora Temprana (TEA) en este segmento, que es de $15,8 \%$. 
En este bloque, también figura China, uno de los países de mayor crecimiento económico constante en los últimos 10 años. Su TEA, es decir, el porcentaje de adultos en proceso de iniciar un negocio o que están gestionando una nueva empresa es de $18,8 \%$, cerca de siete puntos porcentuales por sobre la media del grupo y tres más arriba de Ecuador. Mientras tanto, la economía más poderosa del mundo, Estados Unidos, perteneciente al grupo de economías basadas en la innovación, cuenta con un TEA del 8\%, casi la mitad del de Ecuador y muy por debajo de China.(GEM, 2013)

\begin{tabular}{lcccc}
\hline (en porcentajes) & $\begin{array}{c}\text { Indice de } \\
\text { Actividad } \\
\text { Emprendedora } \\
\text { Temprana }\end{array}$ & $\begin{array}{c}\text { Discontinuidad } \\
\text { de los negocios }\end{array}$ & $\begin{array}{c}\text { Emprendimientos } \\
\text { por necesidad }\end{array}$ & $\begin{array}{c}\text { Emprendimientos } \\
\text { por oportunidad }\end{array}$ \\
\hline Argentina & 14,7 & 6,2 & 47 & 37 \\
\hline Brasil & 15,3 & 4,0 & 39 & 48 \\
\hline Colombia & 22,4 & 7,1 & 34 & 45 \\
\hline Chile & 14,9 & 6,4 & 25 & 42 \\
\hline Ecuador & 15,8 & 6,0 & 32 & 43 \\
\hline Panamá & 9,6 & 1,4 & 24 & 59 \\
\hline Perú & 20,9 & 7,1 & 28 & 26 \\
\hline R. Dominicana & 17,5 & 12,9 & 34 & 57 \\
\hline Uruguay & 12,2 & 4,9 & 22 & 55 \\
\hline Estados Unidos & 8,0 & 3,4 & 23 & 29 \\
\hline China & 18,8 & 6,6 & 48 & 26 \\
\hline
\end{tabular}

Figura 1. Fuente: Tasa Emprendedora a nivel mundial, (GEM, 2013)

El estudio socioeconómico presentado por GEM, permite un enfoque ampliado de la realidad del emprendimiento en el Ecuador desde las diversas áreas de impacto que se derivan del emprender. El emprendimiento según sus formas de desempeño y acción, demanda un, análisis de evolución como aspecto económico y social, para luego estudiarlo desde diversas perspectivas, lo que permite determinar los campos del conocimiento aún no abordado.

En función del contexto del emprendimiento en el Ecuador, los conceptos citados de emprendedor anteriormente e identificadas las perspectivas de estudio; Fonrouge (2002), propone cuatro perspectivas de análisis estas son, la comportamental, la psicológica o cognitiva, la económica y la de procesos. Asociando lo antes expuesto el emprendedor como sujeto de gestión de los emprendimientos, puede ser analizado desde varias perspectivas que en esta ocasión se analizará la perspectiva psicológica o cognitiva, considerando al individuo con capacidad para generar interacciones entre los elementos llamados sociedad, economía en su conjunto de actores y el desempeño individual.

\section{Comunicación}

"Una gestión eficaz de la comunicación puede generar y mejorar la confianza, en un contexto dado. La comunicación tiene el poder de influir en los individuos y la capacidad de generar y mantener la confianza, así como de perderla, cuando hay incongruencias entre lo se dice y hace, o cuando los mensajes se perciben como promesas inalcanzables o con tintes manipuladores."(Viñaras, 2013:61)

Por ello la comunicación se convierte en móvil de transferencia de información en la que se fundamenta la confianza en un determinado contexto, dependiendo de los contenidos y la forma en que ocurra el proceso comunicacional. 
Viñaras (2013), cita a Rafael Alberto Pérez, recordando la importancia de la comunicación estratégica y señala la importancia de la comunicación en el ser humano, en sus opiniones y actos, por ello tiene la capacidad de influir en los demás. La comunicación tiene el poder de influir en los demás, aunque esta influencia sea recíproca y esencial en las relaciones humanas: "La interacción comunicativa en concreto, es un proceso que lleva a ejercer influencia sobre las acciones y las perspectivas de los individuos"(Pérez, 2008:427)

Adicionalmente, Viñaras (2013) citando a Grunig, (1984: 56), “define el enfoque sistémico de la comunicación y las relaciones públicas, y a partir de la Teoría General de Sistemas, las organizaciones se consideran "sistemas", concretamente sistemas abiertos en constante interacción con su entorno, constituido por múltiples subsistemas interdependientes pero coordinados entre sí." (Bertalanffy, 1989)

En este contenido las organizaciones generan diversas relaciones de reciprocidad en aspectos como: político y legales, económicos, sociales, tecnológicos competitivos; estas conexiones tienen como eje de desempeño a la comunicación entre la organización y los actores del entorno. Viñaras (2013)

Así Viñaras (2013), cita Van Riel (1997), el autor principal de internacional de Comunicación Corporativa identifica como una actitud, la naturaleza de los objetivos de la comunicación corporativa: "Los resultados anticipados de la representación son los cambios deseados por la empresa a nivel cognitivo, afectivo y conativo, en aquellos públicos objetivo con los que se pretende establecer y mantener una relación". Adicionalmente, se reconoce a la comunicación como el proceso de transferencia de información para provocar las interacciones sociales en las organizaciones a nivel interno y externo.

Agrega (García Cruz, Valle Cabrera, \& Bou Llusar, 2014), de su investigación empírica, y publicada en AEDEM, para el análisis del comportamiento del directivo hacia el profesor, analiza antecedentes como efectividad en la comunicación, empowerment y valores compartidos, además analiza las consecuencias como comportamiento organizacional ciudadano e intención de permanecer en la organización. En esta investigación se definió y fundamentó en el factor de la efectividad de la comunicación en tres dimensiones y sub clases, determinando valores altos de correlación.

Tabla 3.

\begin{tabular}{|c|c|c|}
\hline & EFECTIVIDAD DE LA COMUNICACIÓN (Mohr y Speckman, 1994) & $\alpha$ \\
\hline & $\begin{array}{l}\text { Calidad de la Comunicación } \\
\end{array}$ & 0.954 \\
\hline ECC1 & Inoportuna/Oportuna & \\
\hline ECC2 & Inexacta/Exacta & \\
\hline ECC3 & Inadecuada/Adecuada & \\
\hline ECC4 & Incompleta/Completa & \\
\hline ECC5 & No creible/Creible & \\
\hline & $\begin{array}{c} \\
\end{array}$ articipación & 0,931 \\
\hline ECP1 & Solicita mis consejos y recomendaciones & \\
\hline ECP2 & Participo con él/ella para establecer objetivos y pronósticos & \\
\hline ECP3 & Le ayudo a planificar las actividades & \\
\hline ECP4 & Solicita mis sugerencias & \\
\hline & Compartir Información & 0,885 \\
\hline ECCl & Comparto todo tipo de información con él/ella & \\
\hline $\mathrm{ECC} 2$ & Le informo por adelantado de las necesidades de cambio & \\
\hline ECC3 & Nos proporcionamos cualquier información que pueda ser ủtil a la otra parte & \\
\hline ECC4 & Cada parte informa a la otra de los acontecimientos que les puedan afectar & \\
\hline
\end{tabular}

Fuente: Extracto de Tabla 2, escala e indicadores usados para medir la variable (García Cruz et al., 2014) 
Estos datos contribuyen al cumplimiento del objetivo del artículo de (García Cruz et al., 2014), que es: "Analizar la relación interpersonal entre el docente y subordinado desde los diferentes factores y su relación con la confianza".

Por otra parte, contribuye a la verificación de la hipótesis de investigación "Factores que generan confianza y explican el comportamiento en contextos donde el intercambio es esencialmente económico ¿se comportan de igual forma cuando el intercambio de naturaleza es social? H1. En un contexto de intercambio social como el que tiene lugar en la universidad se da mucha relación positiva entre la efectividad de la comunicación eficiente entre las partes y el nivel de confianza establecida entre ellos. (Se tomó solo la primera hipótesis y su análisis por la interrelación con el tema de investigación propuesto.)",(García Cruz et al., 2014:64 ) utilizando un método empírico, "cuantitativo, por la autonomía con la que se llevan a cabo las relaciones interpersonales de dependencia en la Universidad. Se seleccionó una muestra de un censo de población, se realizó un análisis factorial confirmatorio y modelo de estructuras de covarianza."

Estos resultados confirman que efectividad de la comunicación, depende de la calidad de comunicación, participación y compartir información, con estos datos se confirma que la confianza es un elemento intangible de cohesión social, que se gestión en función de la comunicación e información en un contexto determinado, (Echeverría, 2000; Viñaras, 2013), es decir a mayor comunicación mayor confianza.

\section{Confianza}

Echeverría (2000:65), analiza a la empresa en la actualidad como “organizaciones que cada vez más se orientan hacia la participación, la autogestión y el trabajo en equipo, entender el trabajo no manual como una red de conversaciones que se constituyen en una interrelación de lenguaje y emociones implica un marco teórico único para entender las relaciones entre los individuos en el interior y exterior de dichas organizaciones. De ese marco teórico se derivan una serie de herramientas que el gerente y el directivo pueden aplicar para incidir de forma directa en la productividad del trabajador no manual, enriqueciendo el ambiente de trabajo y propiciando un mayor bienestar para las personas."

La finalidad de Echeverría (2000), es entender el giro que han dado las empresas en décadas anteriores, respeto a las de la actualidad; es decir que las empresas anteriormente se desarrollaban en ambientes de miedo y amenaza, las actuales se desempeñan en ambientes de confianza y autonomía responsable. "La confianza, en lugar del miedo, se convierte en la emocionalidad que regula las interacciones entre los individuos en el nuevo modelo organizacional que analiza. El control social de los intercambios entre individuos exige la confianza como parámetro por el cual se rigen las relaciones y los intercambios sociales"(Echeverría, 2000:76)

Las organizaciones en la actualidad propenden a la auto organización y adaptación a los entornos cambiantes, pese a ello y la evolución que han sufrido, el miedo aún continua presente en el desempeño de los empleados que no logran desarrollar confianza en las capacidades que poseen, puesto que hoy día la innovación y procesos de mejoramiento lo demandan.(Echeverría, 2000) De esta manera en la actualidad se ha integrado a la confianza como aquel elemento para el desempeño y relaciones desde la dimensión psicológica o cognitiva. 
Desde esta dimensión la confianza está integrada por tres componentes, el cognitivo, el afectivo y el conductual, (Viñaras, 2013), el primero se refiere al conocimiento que surge de la experiencia y el aprendizaje formal, ello se constituye en información pre adquirida que permite el confiar o no, el segundo se define por las emociones, sentimientos que deben ser controladas para mantener la confianza y el tercero "Se refiere a la probabilidad o tendencia de que un individuo realice una acción específica o se comporte de una determinada manera" (Schiffman \& Lazar, 2010: 259) citado por (Viñaras, 2013).

Del análisis bibliográfico desarrollado por (Viñaras, 2013), propone algunas estrategias y tácticas de comunicación, desde las tres dimensiones sicológicas para generar confianza.

\begin{tabular}{|c|c|c|c|}
\hline & \multicolumn{3}{|c|}{ Estratepias y tócticos de comunicación } \\
\hline 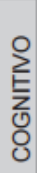 & $\begin{array}{l}\text { Aumentar la cantidad de } \\
\text { información sobre la capacidad } \\
\text { de las instituciones }\end{array}$ & $\begin{array}{l}\text { Reforzar la credibilidad } \\
\text { de la institución y sus } \\
\text { portavoces. } \\
\text { Elegir portavoces que } \\
\text { gocen de credibilidad. } \\
\text { Mejorar la transparencia }\end{array}$ & $\begin{array}{l}\text { Reforzar los atributos } \\
\text { sobre la capacidad de la } \\
\text { institución }\end{array}$ \\
\hline 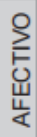 & $\begin{array}{l}\text { Lenguaje más próximo al } \\
\text { ciudadano. } \\
\text { Portavoces empáticos }\end{array}$ & $\begin{array}{l}\text { Crear canales de } \\
\text { comunicación que permitan } \\
\text { bidireccionalidad }\end{array}$ & $\begin{array}{l}\text { Compartir y comunicar a } \\
\text { los ciudadanos principios } \\
\text { y valores que creen } \\
\text { empatia adecuados a la } \\
\text { situación de crisis }\end{array}$ \\
\hline 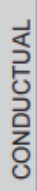 & $\begin{array}{l}\text { Recordar la capacidad de las } \\
\text { instituciones en el pasado }\end{array}$ & $\begin{array}{l}\text { Cuidar más las experiencias } \\
\text { del ciudadano con la } \\
\text { institución }\end{array}$ & $\begin{array}{l}\text { Premiar/agradecer la } \\
\text { conducta positiva hacia } \\
\text { la organización }\end{array}$ \\
\hline
\end{tabular}

Figura 2. Fuente: Cuadro Resumen ejemplos propuestas de estrategias de comunicación para generar confianza (Viñaras, 2013)

Esta propuesta de Viñaras (2013), responde de alguna forma las preguntas de investigación de este artículo son: ¿Es la Confianza una actitud del individuo que requiere estrategias específicas de comunicación? ¿Qué diferencias y similitudes tiene con otros intangibles como la imagen y la reputación? ¿Cuáles son las estrategias de comunicación más adecuadas?, alcanzando el objetivo de este artículo que es "Proponer estrategias de comunicación adaptadas al objetivo actitudinal de recuperar la Confianza, para lo que como objetivo previo se necesita conocer en profundidad el término", a través del método de investigación descriptivo, utilizando fuentes bibliográficas del concepto de Confianza como actitud, tomando como referencia la Psicología social, cognitiva y la Psicología del consumidor, así como la referencia a bibliografía sobre comunicación, relaciones públicas y estrategia.

\section{Emprendimiento, Comunicación y Confianza}

Los emprendimientos son unidades económicas de bajo impacto en la economía del Ecuador por la generación de empleo y el tiempo de supervivencia de los mismos; sobre todo por la gestión individual de carácter sicológico cognitivo. Por otra parte se encuentra la comunicación como aquel flujo de información que moviliza los sistemas sociales en este caso los emprendimientos en las relaciones que mantiene con los grupos de interés como los clientes, proveedores, competidores y la política pública. 
La Comunicación Corporativa son "Los resultados anticipados de la representación son los cambios deseados por la empresa a nivel cognitivo, afectivo y conativo, en aquellos públicos objetivo con los que se pretende establecer y mantener una relación". (Viñaras, 2013), este concepto aplicado al emprendimiento; define desde el campo disciplinar aplicado las categorías de desempeño de este sector de la Economía en el Ecuador. Por otra parte la confianza es un elemento de construcción social, que influye en el rol del individuo y ciudadano. Gordon (2005), manifiesta que "las teorías de la elección racional definen a la confianza como el conjunto de expectativas racionales, basadas en la comprensión del interés del otro con respecto al propio y en cálculos que sopesan los costos y beneficios de ciertos cursos de acción de quien confía o de la persona en quien se confía."

La teoría de la acción racional, (Coleman, 1990) considera que la confianza es el sustento de las relaciones de reciprocidad en que se basa el capital social, de ahí el carácter que reviste este como recurso inmerso en las relaciones sociales, del que se pueden obtener beneficios individuales (Gordon, 2005)

(Luhmann, 1997) distingue entre confiabilidad y confianza y señala que esta distinción depende de la percepción y de la atribución. Cuando no se consideran cursos alternativos de acción, se está en una situación de confiabilidad, y si las expectativas son defraudadas, se atribuye esta desilusión a una causa o factor externo al actor; en cambio, si se escoge una acción por encima de otras, a pesar de la posibilidad de ser defraudado por la acción de los otros, la situación se define como de confianza.

(Luhmann, 1997) distingue entre confianza personal, basada en la familiaridad y en tomar las cosas como dadas y la confianza sistémica o confianza en el funcionamiento adecuado de ciertos sistemas. La primera forma es producto de la interacción previa o derivada de la membresía en un mismo grupo social. La segunda es propia de un orden social complejo en el que se tiende a perder la familiaridad, y la refuerzan mecanismos que, como la ley, limitan el riesgo de la confianza mal depositada. Estos mecanismos actúan como estructuras que dan seguridad sin necesidad de ser activadas, ya que el uso efectivo de sanciones legales es incompatible con una relación de confianza.

Finalmente, el enfoque sistémico de Luhman (2002), expone la función que cumple la confianza. Para este autor, la confianza es un mecanismo que permite a los actores reducir la incertidumbre a través de adoptar expectativas específicas sobre el comportamiento futuro del otro y predecir en alguna medida sus acciones, de manera rutinaria, no necesariamente producto del cálculo.

\section{Conclusiones y Recomendaciones}

La comunicación es una estrategia generadora de confianza en los emprendimientos, por la capacidad de influencia en los individuos y la capacidad de generar y mantener la confianza, así como de perderla, cuando hay incongruencias entre lo se dice y hace, o cuando los mensajes se perciben como promesas inalcanzables o con tintes manipuladores.(Viñaras, 2013). 
Las investigaciones utilizadas como guía directriz, conllevan metodologías, descriptivas y empíricas de tipo cualitativo y cuantitativo, estas se fundamentaron en la revisión bibliografía y análisis estadístico correlacional, que sustentan una propuesta de estrategias de comunicación adaptadas al objetivo actitudinal de recuperar la confianza. La comunicación explica desde la dimensión sicológica, que es la gestión eficaz para generar y mejorar la confianza, en un contexto determinado, por ello propone algunas estrategias y tácticas de comunicación, desde las tres dimensiones sicológicas para generar confianza como, aumentar la cantidad de información sobre la capacidad de las instituciones, lenguaje más próximo al ciudadano y recordar la capacidad de las instituciones en el pasado.

La comunicación, está integrada por factores que generan confianza y explican el comportamiento en contextos donde el intercambio se gestiona, uno de estos elementos se denomina efectividad de la comunicación que se analizó desde sus tres dimensiones, la calidad de la comunicación, participación y compartir información, alcanzado el $98 \%$ de niveles de correlación con la confianza, lo cual señala la relación directamente proporcional que existe entre la comunicación y la confianza. (Luhmann, 1997) distingue entre confiabilidad y confianza, señala que esta distinción depende de la percepción y de la atribución. Que se fundamenta en la capacidad de administrar la comunicación de acuerdo al grupo objetivo y al contexto de desempeño.

A partir de las conclusiones generales, se pueden proponer, algunas preguntas guía como recomendaciones hacia nuevos procesos investigativos. Estos cuestionamientos profundizarían el tema abordado anteriormente, ¿De qué forma el emprendedor ecuatoriano gestionan el proceso comunicacional para relacionarse con los grupos de interés?, ¿Qué variables afectan el proceso comunicacional entre el emprendedor y los grupos de interés?, ¿La comunicación es una estrategia generadora de confianza en los emprendedores del Ecuador?, Los procesos investigativos futuros responderán que han surgido de la presente investigación.

\section{Bibliografía}

Bertalanffy, L. (1989). General System Theory. Teoría General de Los Sistemas, 311. Retrieved from http://cienciasyparadigmas.files.wordpress.com/2012/06/teoria-general-de-los-sistemas-_fundamentos-desarrollo-aplicacionesludwig-von-bertalanffy.pdf

Casson, M. (2005). Entrepreneurship and the theory of the firm. Journal of Economic Behavior and Organization, 58(2), 327-348. http://doi.org/10.1016/j.jebo.2004.05.007

Claudia Eugenia Toca Torres. (2017). Aportes a la responsabilidad social. Revista Mexicana de Ciencias Políticas Y Sociales, 230, 394-408. Retrieved from file://C:/Users/Lizbeth Suarez/Downloads/SSRN-id2962634.pdf

Coleman, J. S. (1990). Fundamentos de teoría social. (C. de I. Sociológicas, Ed.) (Primera en). MadridEspaña. Retrieved

from https://books.google.com/books?hl=es\&lr=\&id=Hev3oZ1 vqSkC\&pgis=1

Drakopoulou Dodd, S., \& Hynes, B. C. (2011). The impact of regional entrepreneurial contexts upon enterprise education. Entrepreneurship \& Regional Development, (December 2012), 1-26. http://doi.org/10.1080/08985626.2011.566376 
Echeverría, R. (2000). La empresa emergente, la confianza y los desafíos de la transformación. (Granica, Ed.) (Primera Ed). Chile. Retrieved from https://books.google.com/books?hl=es\&lr=\&id=u6j5GhITAOwC\&pgis=1

Fonrouge, C. (2002). Entrepreneur/Manager: deux acteurs d'une même pièce. Retrieved December 21, 2015 , https://scholar.google.com.br/citations?view_op=view_citation\&hl=es\&user=nkTCLkAAAAJ\&citation_for_view=nkTCL-kAAAAJ:qjMakFHDy7sC

García Cruz, J., Valle Cabrera, R., \& Bou Llusar, J. C. (2014). La generación de confianza en un contexto de intercambio social: la relación supervisor-supervisado. Revista Europea de Dirección $Y$ Economía de La Empresa, 23(2), 61-71. http://doi.org/10.1016/j.redee.2012.09.001

GEM, E. (2013). GEM. Retrieved from http://www.espae.espol.edu.ec/wpcontent/uploads/2017/06/emprendedores_senior.pdf

Gordon, S. (2005). Confianza, capital social y desempeño de organizaciones. Retrieved December 21, 2015, from http://www.plataformademocratica.org/Publicacoes/14210_Cached.pdf\#page=39

Luhman, N. (2002). La Teoría de Niklas Luhmann. Retrieved December 21, 2015, from http://www.infoamerica.org/documentos_pdf/luhmann_01.pdf

Luhmann, N. (1997). The Society of Society: The Grand Finale of Niklas Luhmann. Sociological Theory, 18(2), 320-330. http://doi.org/10.1111/0735-2751.00102

Pérez, A. (2008). Estrategias de comunicación. Ariel. Retrieved from https://books.google.com.ec/books/about/Estrategias_de_comunicaci\%C3\%B3n.html?id=Ki5 AXzNODg8C\&pgis $=1$

Rodríguez, A. (2009). Nuevas perspectivas para entender el emprendimiento empresarial. Retrieved December 21, 2015, from http://rcientificas.uninorte.edu.co/index.php/pensamiento/article/viewFile/864/508

Schiffman, L., \& Lazar, L. (2010). Comportamiento del Consumidor. Retrieved from www.pearsoneducacion.net/schiffman

Schumpeter. (1950). Schumpeter, J., Stiglitz, J. (2010). Capitalism, Socialism and Democracy. London: Routledge. New York: Taylor\&Francis. Retrieved from https://www.taylorfrancis.com/books/9781135154752

Senescyt. (2010). Plan Nacional de Ciencia y Tecnología. Retrieved May 19, 2016, from http://www.ilades.edu.ec/publicaciones/Plan Nacional de Ciencia, Tecnolog\%C3\%ADa, Innovaci\%C3\%B3n y Saberes ancestrales.pdf

Viñaras, M. (2013). Estrategias de comunicación para generar confianza. Retrieved May 10, 2016, from http://studyres.es/doc/3416291/estrategias-de-comunicaci\%C3\%B3n-para-generarconfianza 